\title{
ROLE OF DIRECT IMMUNOFLUORESCENCE ON TZANCK SMEAR IN PEMPHIGUS VULGARIS
}

\author{
Arun Jose1, Sheeja S2, Mary Vineetha3 ${ }^{3}$, Sankar $S^{4}$ \\ 1Junior Resident, Department of Pathology, GMC, Kottayam, Kerala, India. \\ ${ }^{2}$ Associate Professor, Department of Pathology, GMC, Kottayam, Kerala, India. \\ ${ }^{3}$ Assistant Professor, Department of Dermatology, Venereology and Leprosy, GMC, Kottayam, Kerala, India. \\ 4 Professor and HOD, Department of Pathology, GMC, Kottayam, Kerala, India.
}

ABSTRACT
BACKGROUND
The Tzanck smear is a simple, sensitive, and rapid test to diagnose pemphigus vulgaris (PV), a life-threatening autoimmune
blistering disorder. The presence of acantholytic cells in cytology is indicative of, but not specific for PV. Hence, a direct
Immunofluorescence (DIF) test to demonstrate immunoglobulin deposits on the acantholytic cells would make the Tzanck test
more specific, and rapid. The DIF smears were compared with DIF on skin biopsies in the same patient to evaluate the diagnostic
efficacy.
Aims and Objectives-To study the expression of IgG and C3 in cytology of pemphigus vulgaris using Tzanck smears and to compare
direct immunofluorescence in Tzanck smears with corresponding perilesional skin biopsies.

\section{MATERIALS AND METHODS}

Study Design- Diagnostic test evaluation.

Study Population- Study was performed on oral scrape smears procured from clinically diagnosed cases of pemphigus vulgaris attending the dermatology department during the study period. (March 2017-August 2018).

Sample Size- 30 .

Sampling Procedure- Continuous sampling.

Study Procedure- DIF for IgG and C3 done on both Tzanck smears and corresponding perilesional skin biopsies.

Analysis-Sensitivity, specificity, positive predictive value and negative predictive value was used to compare immunofluorescence in Tzanck smears with skin biopsies. Non-parametric test (Kendall's tau-b) was used to assess correlation.

\section{RESULTS}

Age group of study population ranges from 14 to 74 years with mean age being 49 years. IgG was positive in $80 \%$ of Tzanck smears and $83 \%$ of skin biopsies. C3 was positive in $60 \%$ of Tzanck smears and $70 \%$ of skin biopsies. Sensitivity, specificity, positive predictive value (PPV), negative predictive value (NPV) of Tzanck smears in the investigation of IgG were (\%) 92, 80, 95, and 66; and C3 were (\%) 76, 77, 88, 58. The correlation coefficient between Tzanck smears and corresponding skin biopsies for IgG and C3 were 0.670 and 0.505 respectively.

\section{CONCLUSION}

There is definite correlation between IgG and C3 staining in Tzanck smears compared to skin biopsy with a correlation coefficient of 0.670 and 0.505 respectively. So DIF on Tzanck smears can be used for presumptive diagnosis of pemphigus vulgaris which avoids the need for biopsy and delay in starting therapy.

\section{KEY WORDS}

Tzanck Smear, Skin Biopsy, IgG, C3, Direct Immunofluorescence.

HOW TO CITE THIS ARTICLE: Jose A, Sheeja S, Vineetha M, et al. Role of direct immunofluorescence on Tzanck smear in pemphigus vulgaris. J. Evolution Med. Dent. Sci. 2019;8(04):254-259, DOI: 10.14260/jemds/2019/55

\section{BACKGROUND}

Pemphigus is a group of autoimmune, intraepithelial, chronic blistering skin diseases characterised by loss of normal intercellular adhesion. It occurs due to the formation of autoantibodies, mainly IgG \& or C3 against epithelial adhesion molecules, desmosomes. ${ }^{1,2}$

'Financial or Other Competing Interest': None.

Submission 20-12-2018, Peer Review 16-01-2019,

Acceptance 22-01-2019, Published 28-01-2019.

Corresponding Author:

Dr. Arun Jose,

Junior Resident,

Department of Pathology,

Government Medical College,

Gandhinagar, P. O. Kottayam-686008,

Kerala, India.

E-mail: josearun420@gmail.com

DOI: $10.14260 /$ jemds $/ 2019 / 55$
There are three major types of pemphigus, which vary in severity-pemphigus vulgaris, pemphigus foliaceus and paraneoplastic pemphigus. ${ }^{1,2}$ Pemphigus vulgaris and foliaceus differ in the level of intraepithelial blisters-in vulgaris suprabasal, and in foliaceus subcorneal bullae are formed. ${ }^{2}$

Pemphigus vulgaris (PV) is the most common pemphigus disorder. It affects mainly middle-aged adults, both sexes equally. It is characterised by autoantibodies against desmoglein-3. It shows a geographical predilectionMediterranean, South Asia and Jewish region are affected more. ${ }^{2}$

Clinical features are cutaneous or mucosal blisters. Oral lesions precede skin lesions in more than $50 \%$ of the patients and presents as blisters which rupture rapidly resulting in painful erosions. Buccal mucosa, lips and soft palate are the most commonly affected sites.2,3,4 Diagnosis of PV is based on clinical, histopathological and immunological correlation.1,2 
Skin biopsy is done for tissue diagnosis ${ }^{3}$. A proper histopathological examination can form the diagnosis in accordance with the location and morphology of blisters. Demonstration of immunoglobulins in the spinous cell junctions by direct immunofluorescence (DIF), from perilesional biopsy (Within $1 \mathrm{~cm}$ of the lesion) is often used for a complete diagnosis of PV. ${ }^{3}$

Immunofluorescence is a histochemical laboratory staining technique used for demonstrating the presence of antibodies bound to antigens in tissues or circulating body fluids. This technique is used to supplement clinical and histopathological findings in PV and other vesiculobullous diseases. They help in the early diagnosis, treatment and monitoring of the disease activity in PV. ${ }^{4}$

Pemphigus vulgaris has a relentless course, unless timely identified and its immunological progression checked, it can lead to a fatal outcome. So early diagnosis of the condition is imperative to prevent complications. DIF can be done both on tissue biopsies and in cytology smears for confirming the diagnosis. ${ }^{5}$
Tzanck smear combined with DIF is used as an effective and simple tool, in the rapid diagnosis of PV.5,6,7 The Tzanck smear has the advantages of being easy to perform, inexpensive, not requiring a specialised laboratory, and causing negligible trauma and discomfort to the patient.7

\section{Objectives of The Study}

To assess the role of direct immunofluorescence (DIF) on Tzanck smear taken from mucosal or cutaneous lesions in pemphigus vulgaris and compare it with DIF done on skin biopsy.

\section{MATERIALS AND METHODS \\ Study Design}

Diagnostic test evaluation.

\section{Study Period}

18 months (March 2017-August 2018).

\section{Study Setting}

Department of Pathology, Govt. Medical College, Kottayam.

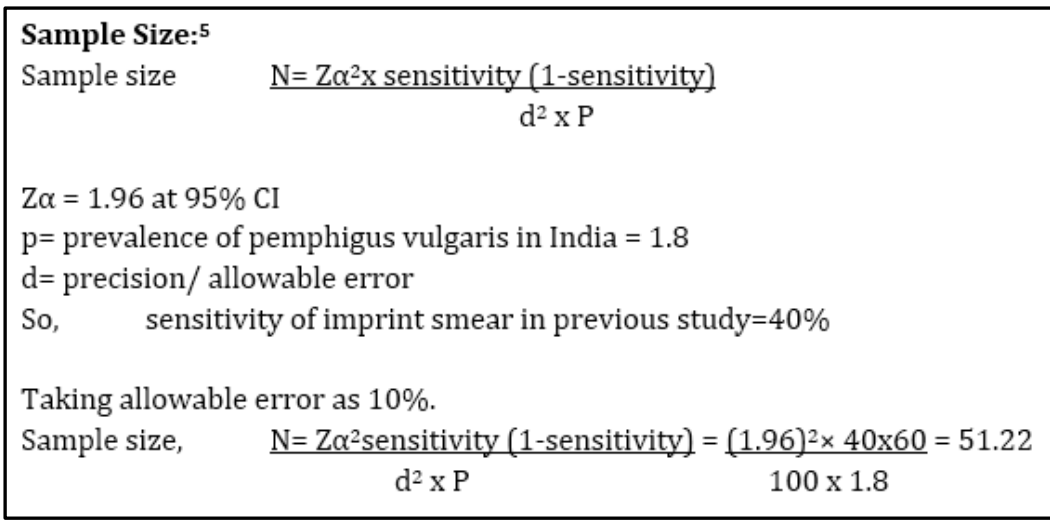

Taking sample size as 51.

Calculated sample size is 51 . As the annual number of patients newly diagnosed as pemphigus per year is below 30 in the Department of Dermatology, Government Medical College Kottayam, this study included all newly diagnosed cases of pemphigus.

\section{Inclusion Criteria}

All clinically diagnosed cases of pemphigus vulgaris were included following a histopathological confirmation.

\section{Exclusion Criteria}

1. Previously treated pemphigus vulgaris patients.

2. Other pemphigus group of diseases.

3. Patients who are not willing to take part in the study.

Clinically suspected cases of PV attending the skin OPD were evaluated. Study was performed from oral scrape smears procured from clinically diagnosed cases of pemphigus vulgaris attending the dermatology department during the study period. Two scrape smears were taken from oral erosions and air dried and sent immediately to the
Department of Pathology. One was stained with MayGrunwald-Giemsa (MGG) stain and detailed cytological analysis done. If oral/mucosal lesions were not present skin erosions are scraped, smeared and evaluated.

The other air-dried smear for DIF staining was stained with fluorescein conjugated rabbit antihuman IgG and with C3 (Dako) for 30 min. Then the smear was rinsed in PBS solution three times for $5 \mathrm{~min}$ each, mounted in buffered glycerol and examined immediately under the immunofluorescence microscope. If the smears were positive for immunofluorescence the pattern of staining by the acantholytic cells were noted. The skin biopsy specimens of these patients were received in $10 \%$ formalin solution and DIF on skin biopsy was done. Results of DIF on Tzanck smear were correlated with DIF on biopsy, which is the gold standard. Histological diagnosis was made and correlated with Tzanck smear findings.

Written informed consent from each patient was taken prior to the procedures.

\section{Data Management and Analysis}

The data was entered in Microsoft excel and further statistical analysis was done using SPSS software (version 20). 


\section{Statistical Methods}

1. Sensitivity, specificity, positive predictive value and negative predictive value of Tzanck smears in the assessment of IgG and C3 immunofluorescence was compared with the same in histopathology.

2. Non-parametric test (Kendall tau b) for-

- Correlation of IgG fluorescence in Tzanck smear as compared to skin biopsy.

- Correlation of C3 fluorescence in Tzanck smears as compared to skin biopsy.

The level of significance was indicated by correlation coefficient (Between 0 and 1).

\section{RESULTS}

Diagnostic test evaluation was done on 30 cases of pemphigus vulgaris presented to Department of Pathology, Government medical college, Kottayam during the study period of 18 months (March 2017-August 2018).

- DIF for IgG and C3 were performed on the Tzanck smears from these cases and the results were compared with the DIF for IgG and C3 done on perilesional skin biopsies.

- The mean age of the present study population was 49 and minimum age was

14 years and maximum was 74 years.

- $\quad 53 \%$ of the study population were females.

- Majority of study population (70\%) had duration of illness not exceeding 6 months.

- Oral mucosa was the initial site involved in majority of cases (70\%) followed by skin (7\%).

- Initial lesions were erosions in $67 \%$ of patients and vesicle in the remaining cases (33\%).

- $\quad$ The disease process was generalized in $63 \%$ of cases and localized in $37 \%$ cases.

- Skin lesions were present in $80 \%$ of the cases, with predominant trunk involvement.

- $\quad$ Erosions were predominant lesion $(80 \%)$ followed by vesicles (20\%).

- Oral mucosa was involved in $96 \%$ of cases, genital mucosa in 40\%, nasal mucosa in 30\%.

- Tzanck smear cytology showed acantholytic cells in all the cases with neutrophils as the predominant inflammatory cell.

- Histopathological evaluation of skin biopsies showed suprabasal clefting in all the cases with many showing acantholytic cells and row of tombstone appearance.

- DIF on perilesional skin biopsies showed fishnet positivity for IgG in $83 \%$ of cases and for C3 in $70 \%$ of cases.

- DIF on Tzanck smears showed fishnet positivity in epithelial keratinocytes for IgG in $80 \%$ of cases and for C3 in $60 \%$ of cases.

- $\quad$ Sensitivity for IgG was $92 \%$ and for C3 was $76 \%$, on Tzanck smear.

- $\quad$ Specificity for IgG was $80 \%$ and for C3 was $77 \%$, on Tzanck smear.

- $\quad$ Positive predictive value for IgG was 95\% and for C3 was $88 \%$, on Tzanck smear.

- $\quad$ Negative predictive value for IgG was $66 \%$ and for C3 was $58 \%$, on Tzanck smear.
- The correlation coefficient between the expression of IgG and C3 on Tzanck smear and that on the skin biopsy was 0.670 for IgG and 0.505 for C3.

- $\quad P$ value for IgG was $<0.0004$ and for C3 was $<0.008$, which is statistically significant.

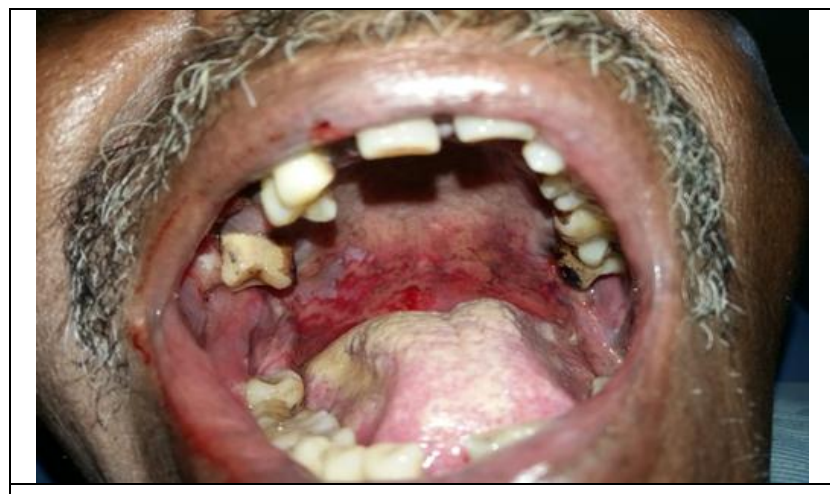

Figure 1. Oral Lesions-Pemphigus Vulgaris

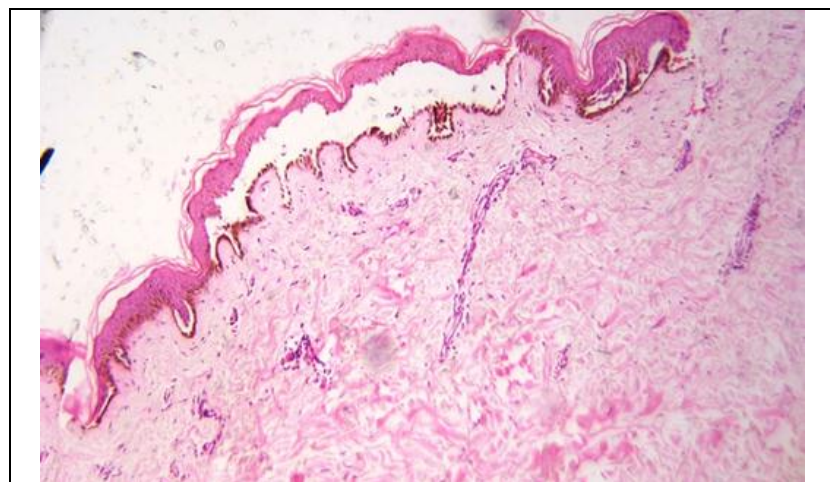

Figure 2. Histopathology-Suprabasal Bullae Showing Tombstone Appearance- H\&E- $100 \mathrm{X}$

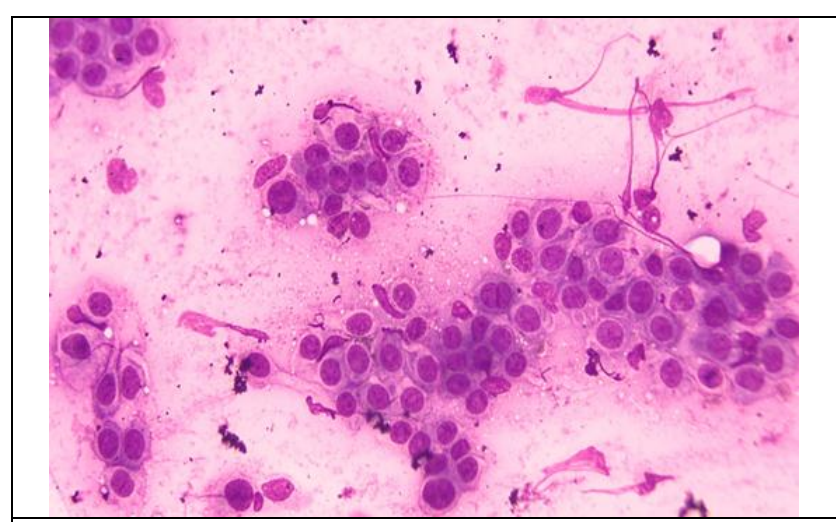

Figure 3. Oral Tzanck Smear Showing Acantholytic CellsGiemsa- 400X

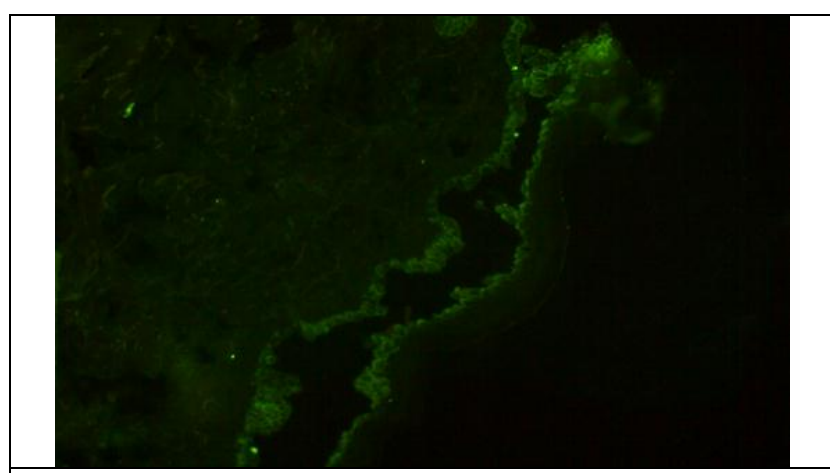

Figure 4. DIF Skin Biopsy- Fishnet Positivity for IgG (200X) 


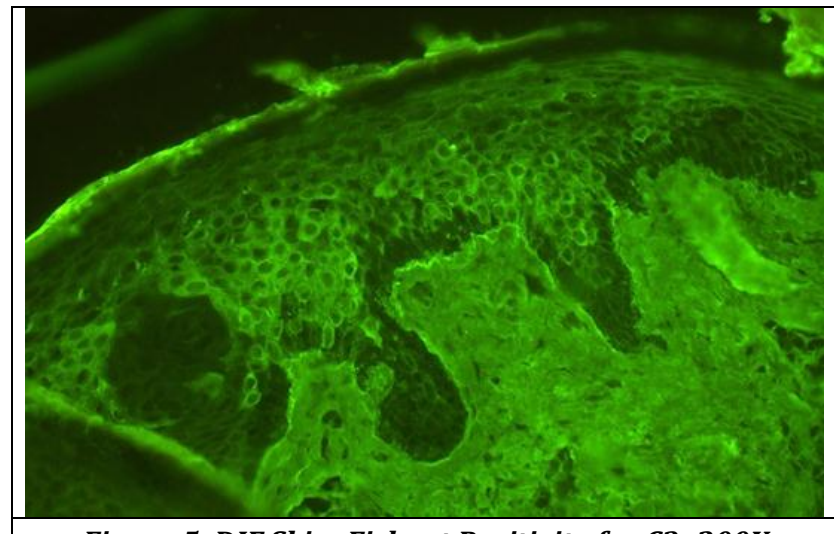

Figure 5. DIF Skin- Fishnet Positivity for C3-200X

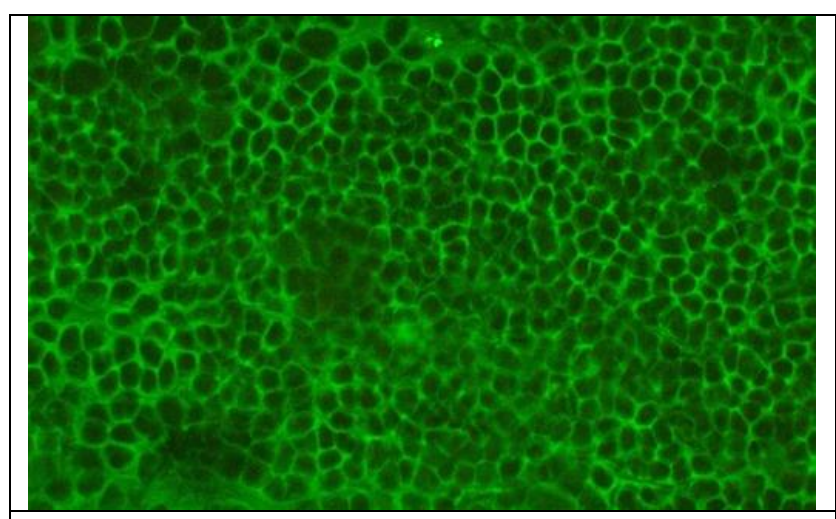

Figure 6. DIF Tzanck Smear- Fishnet Positivity for C3-200X
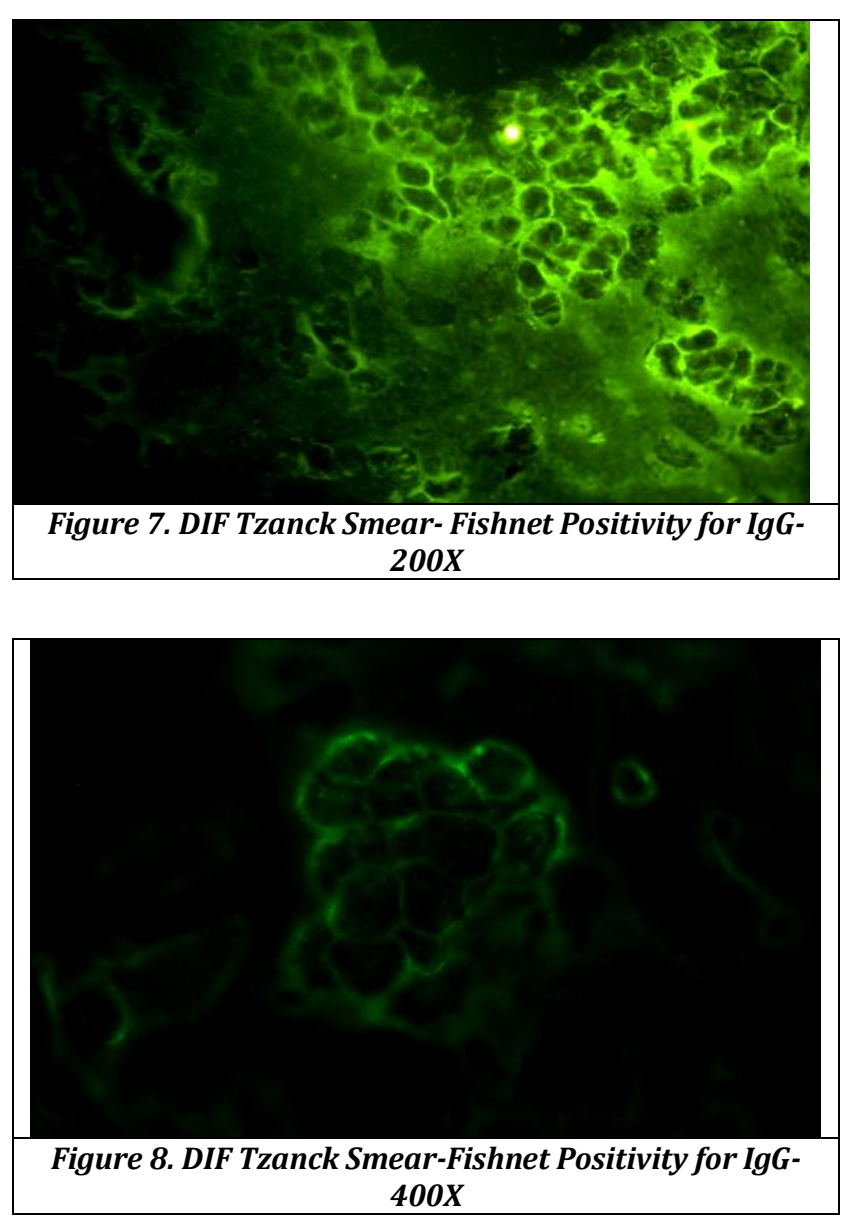

\begin{tabular}{|c|c|}
\hline Study & Mean Age (Years) \\
\hline Present Study & 49 \\
\hline Aithal et $\mathrm{al}^{5}$ & 47.35 \\
\hline${\text { Yaeen et }{ }^{6}}^{6}$ & 35.1 \\
\hline \multicolumn{2}{|c|}{ Table 1. Comparison of Mean Age with Other Studies } \\
\hline
\end{tabular}

In the present study the study population consisted of 16 females (53.33\%) and 14 males (46.67\%). The slight female preponderance is comparable to study conducted by Srinath et al $^{1}$. Study conducted by Aithal et $\mathrm{al}^{5}$ showed equal distribution among both sexes.

\begin{tabular}{|c|c|c|}
\hline Study & Males (\%) & Females (\%) \\
\hline Present Study $^{\text {Srinath et al }}{ }^{1}$ & 46.67 & 53.33 \\
\hline${\text { Aithal et } \mathrm{l}^{5}}^{5}$ & 46.67 & 53.33 \\
\hline \multicolumn{3}{|c|}{ Table 2. Comparison of Gender Distribution with Other } \\
Studies \\
\hline
\end{tabular}

In the present study $70 \%$ of cases presented within 6 months of onset of disease. This is comparable to the study conducted by Aithal et $\mathrm{al}^{5}$ in which $75 \%$ of cases presented within 3 months of onset of disease.

In the present study $70 \%$ of cases had oral mucosa as their initial site of involvement. This is comparable to the study conducted by Suliman et $\mathrm{al}^{2}$ in which $57 \%$ of cases had oral mucosa as their initial site of involvement.

Skin was involved in $80 \%$ of cases in the present study. This is comparable to studies conducted by Srinath et al. ${ }^{1}$ Suliman et $\mathrm{al}^{2}$ and Aithal et al, 5 which reported skin involvement in $86 \%, 85 \%$ and $75 \%$ of cases, respectively.

\begin{tabular}{|c|c|}
\hline Study & Skin Involvement (\%) \\
\hline Present Study & 80 \\
\hline Srinath et al ${ }^{1}$ & 86.67 \\
\hline Suliman et al ${ }^{2}$ & 85.71 \\
\hline Aithal et al $^{5}$ & 75 \\
\hline \multicolumn{2}{|c|}{$\begin{array}{l}\text { Table 3. Comparison of Skin Involvement with Other } \\
\text { Studies }\end{array}$} \\
\hline
\end{tabular}

The predilection of various site involvement in the present study were as follows-Trunk $(73 \%)$, extremities (66\%), face (63\%), scalp (60\%).

This is comparable to study conducted by Srinath et al ${ }^{1}$ which showed involvement as follows- trunk (73\%), extremities (46\%), face (53\%) and scalp (33\%).

This is also comparable to a study conducted by Suliman et $\mathrm{al}^{2}$ which reported extremities and trunk as the most common sites of involvement followed by scalp.

\begin{tabular}{|c|c|c|c|c|}
\hline Study & Trunk & extremities & face & Scalp \\
\hline Present Study & 73 & 66 & 63 & 60 \\
\hline Srinath et al 1 & 73 & 46 & 53 & 33 \\
\hline Table 4. Comparison of Various Site Involvement with \\
Different Studies (\%) \\
\hline
\end{tabular}

Erosions (80\%) were predominant lesions in the present study followed by vesicles, bullae and pustules. This is comparable to study conducted by Suliman et $\mathrm{al}^{2}$ which reported erosions as the predominant lesions followed by ulcers and vesicles. 
Oral mucosa was the most common site involved in the present study with $96 \%$ of the patients having oral lesions. This is comparable to studies conducted by Srinath et al ${ }^{1}$ and Suliman et $\mathrm{al}^{2}$ which reported oral mucosal involvement in $100 \%$ and $90 \%$ of cases, respectively.

\begin{tabular}{|c|c|}
\hline Study & Oral Involvement \\
\hline Present Study & $96 \%$ \\
\hline Srinath et al ${ }^{1}$ & $100 \%$ \\
\hline Suliman et al $^{2}$ & $90 \%$ \\
\hline \multicolumn{2}{|c|}{$\begin{array}{l}\text { Table 5. Comparison of Oral Involvement with Various } \\
\text { Studies }\end{array}$} \\
\hline
\end{tabular}

The characteristic cytological finding in Tzanck smear in cases of pemphigus vulgaris are the presence of acantholytic cells. Cytological examination of Tzanck smears, by Giemsa stain is in itself a very sensitive and rapid test to diagnose PV. It is also an easier technique when compared with biopsy, to sample multiple sites as well as poorly accessible sites like the retro molar area, but the findings on Tzanck alone are not pathognomonic for $\mathrm{PV}$, because the characteristic acantholytic cells could also be observed in other subtypes of pemphigus.

In the present study acantholytic cells were present in Tzanck smears in all 30 cases (100\%). This was comparable to the studies conducted by Srinath et $\mathrm{al}^{1}$ and Durdu et al ${ }^{8}$, both of which reported $100 \%$ positivity for acantholytic cells, but the study done by Shailaja et al 7 showed only $50 \%$ positivity for acantholytic cells.

\begin{tabular}{|c|c|}
\hline Study & Presence of Acantholytic Cells \\
\hline Present Study & $100 \%$ \\
\hline Srinath et al ${ }^{1}$ & $100 \%$ \\
\hline Durdu et al8 & $100 \%$ \\
\hline Shailaja et al7 & $50 \%$ \\
\hline \multicolumn{2}{|c|}{$\begin{array}{l}\text { Table 6. Comparison of Cytological Positivity of Tzanch } \\
\text { Smears }\end{array}$} \\
\hline
\end{tabular}

In the present study neutrophils were the predominant inflammatory component in Tzanck smears. This is comparable to a study conducted by Aithal et $\mathrm{al}^{5}$ which also reported neutrophils as the predominant inflammatory component in Tzanck smears.

Histopathological examination of skin biopsy revealed suprabasal cleft in $100 \%$ of cases and acantholytic cells in $90 \%$ of cases in the present study. This is comparable to studies conducted by Srinath et al ${ }^{1}$ and Kabir et al ${ }^{9}$ which showed suprabasal cleft with acantholytic cells in $100 \%$ and $87 \%$ of cases, respectively. Also comparable to study conducted by Suliman et $\mathrm{al}^{2}$ which showed suprabasal cleft in $90 \%$ of cases.

\begin{tabular}{|c|c|c|}
\hline Study & $\begin{array}{c}\text { Suprabasal Cleft } \\
\text { (\%) }\end{array}$ & $\begin{array}{c}\text { Acantholytic Cell } \\
\text { (\%) }\end{array}$ \\
\hline Present Study & 100 & 90 \\
\hline Srinath et $\mathrm{al}^{1}$ & 100 & 100 \\
\hline Kabir et al $^{9}$ & 87 & 87 \\
\hline Suliman et al & \\
\hline \multicolumn{2}{|c|}{ Table 7. Comparison of Histopathological Findings with } \\
Other Studies \\
\hline
\end{tabular}

Even though the Tzanck smears are highly sensitive, they are not specific for pemphigus. To improve the specificity, DIF is performed on Tzanck smears which makes it a useful diagnostic tool in the early diagnosis of pemphigus. Present study showed DIF positivity for IgG in 24/30 Tzanck smears studied (80\%). In a study done by Kabir e tal ${ }^{9}$ DIF on Tzanck smears showed positivity for IgG in 13/15 cases (86\%). Study done by Durdu et al ${ }^{8}$ showed IgG positivity in $14 / 14$ Tzanck smears (100\%). Proportion of clinically diagnosed cases of pemphigus vulgaris showing positivity for IgG by DIF on Tzanck smears in studies conducted by Acosta et $\mathrm{al}^{10}$ and Varma et $\mathrm{al}^{11}$ were $76 \%$ and $77 \%$ respectively.

\begin{tabular}{|c|c|}
\hline Study & IgG Positivity \\
\hline Present Study & $80 \%$ \\
\hline Kabir et al ${ }^{9}$ & $86 \%$ \\
\hline Durdu et al 8 & $100 \%$ \\
\hline Acosta et al ${ }^{10}$ & $76 \%$ \\
\hline Varma et al ${ }^{11}$ & $77 \%$ \\
\hline \multicolumn{2}{|c|}{$\begin{array}{l}\text { Table 8. Comparison of DIF Positivity for IgG On Tzanck } \\
\text { Smears }\end{array}$} \\
\hline
\end{tabular}

\section{DISCUSSION}

The present study was conducted on 30 cases of Pemphigus Vulgaris patients who presented in the Department of dermatology and whose Tzanck smear samples and perilesional skin biopsies were concurrently received in the Department of Pathology Government medical college Kottayam during the period from March 2017-August 18. DIF was done on Tzanck smear for IgG and C3 and it was compared with DIF done on their corresponding histopathology sections.

The mean age of the present study population was 49 . Minimum age was 14 years and maximum was 74 years. Majority belonged to age groups of 30-40 and 40-50 with 6 patients, i.e., $20 \%$ each. Mean age is comparable to study conducted by Aithal et $\mathrm{al}^{5}$ and Yaeen et al. ${ }^{6}$

\section{CONCLUSION}

1. DIF on Tzanck smear from mucosal or skin lesion showed a positivity of $80 \%$ for IgG and $60 \%$ for C3.

2. The corresponding perilesional skin biopsies on DIF showed a positivity of $83 \%$ for IgG and $70 \%$ for $\mathrm{C} 3$.

3. Hence the correlation coefficient between the expression of IgG and C3 on Tzanck smear and that on the skin biopsy was assessed and was found to be 0.670 for IgG and 0.505 for $\mathrm{C} 3$.

Based on the correlation coefficient, DIF on Tzanck smear can be considered a reasonably good supportive diagnostic test for pemphigus vulgaris and may be recommended after larger series of similar studies are performed.

\section{ACKNOWLEDGMENT}

I express my sincere and heartfelt gratitude to Dr. Sankar S., Professor and Head of Department of Pathology, Dr. Sheeja S, my guide and Dr. Mary Vineetha, my co-guide and Dr. Ginju, Dr. Geethanjali and Mr. Josin Mathew for extending their invaluable help in furnishing my dissertation. 


\section{REFERENCES}

[1] Kambil SM, Madavamurthy P. Immunobullous disorders: clinical histopathological and immunofluorescence study of thirty-six cases. Muller J Med Sci Res 2014;5(2):134-8.

[2] Suliman NM, Astrom AN, Ali RW, et al. Clinical and histological characterization of oral pemphigus lesions in patients with skin diseases: a cross sectional study from Sudan. BMC Oral Health 2013;13:66.

[3] Arpita R, Monica A, Venkatesh N, et al. Oral pemphigus vulgaris: case report. Ethiopian Journal of Health Sciences 2015;25(4):367-72.

[4] Mohan KH, Pai S, Rao R, et al. Techniques of immunofluorescence and their significance. Indian J Dermatol Venereol Leprol 2008;74(4):415-9.

[5] Aithal V, Kini U, Jayaseelan E. Role of direct immunofluorescence on Tzanck smears in pemphigus vulgaris. Diagnostic Cytopathology 2007;35(7):403-7.

[6] Yaeen A, Ahmad QM, Farhana A, et al. Diagnostic value of Tzanck smear in various erosive, vesicular and bullous skin lesions. Indian Dermatol Online J 2015;6(6):381-6.
[7] Prabhala S, Deshpande AK, Reddy M, et al. Study of Tzanck smears over a period of six months. Journal of Evidence Based Medicine and Healthcare 2015;2 (10):1365-71.

[8] Kabir AK, Kamal M, Choudhury AM. Clinicopathological correlation of blistering diseases of skin. Bangladesh Med Res Counc Bull 2008;34(2):4853.

[9] Durdu M, Baba M, MD, Seckin D. The value of Tzanck smear test in diagnosis of erosive, vesicular, bullous and pustular skin lesions. J Am Acad Dermatol 2008;59(6):958-64.

[10] Acosta AE, Hietanen J, Ivanyi L. Direct immunofluorescence on cytological smears in oral pemphigus. Br J Dermatol 1981;105(6):645-51.

[11] Verma KK, Khaitan BK, Singh MK. Antibody deposits in Tzanck smears in pemphigus vulgaris. J Cutan Pathol 1993;20(4):317-9. 